\title{
Groundwater Oxygen Anomaly Related to the 2016 Kumamoto earthquake in Japan
}

Yuji Sano', Satoki Onda', Takanori Kagoshima', Toshihiro Miyajima', Naoto Takahata', Tomo Shibata², Chika Nakagawa ${ }^{3}$, Tetsuji Onoue ${ }^{4}$, and Daniele L. Pinti $^{5}$

'Atmosphere and Ocean Research Institute, University of Tokyo,ysano@aori.u-tokyo.ac.jp, Japan

Institute for Geothermal Sciences, Kyoto University, Japan

${ }^{3}$ Department of Earth and Environmental Sciences, Kumamoto University, Japan

${ }^{4}$ Department of Earth and Planetary Science, Kyushu University, Japan

${ }^{s}$ GEOTOP and Research Center on Earth Ssytem Dynamics, University of Quebec at Montreal, Canada

Geochemical precursor of earthquake has attracted the attention of researchers worldwide, because they are not entirely unexpected. Hydrogeochemical changes including stable isotopes were reported before and after a major earthquake in Iceland. Even though these anomalies are promising in future mitigation of earthquake, more data are required to confirm the predictability. We present here data of oxygen and hydrogen isotope variations in groundwater related to earthquake in Japan.

We have collected commercial bottled mineral water samples covering a couple of years in M7.3 earthquakes occurred in Kumamoto, Southwest Japan. They were filtered and sealed in polyethylene terephthalate bottles and distributed on the market. Hydrogen $(2 \mathrm{H} / \mathrm{H})$ and oxygen $\left({ }^{18} \mathrm{O} /{ }^{\circ} \mathrm{O}\right)$ isotopes of water samples were measured by a cavity ring-down spectroscopy (L2120-I Analyzer, PICARRO Co. Ltd) without any chemical preprocessing. Observed hydrogen and oxygen isotopic ratios were calibrated against our in-house standard and converted into the conventional V-SMOW unit, expressed as per mil (\%o).

At the 2016 Tottori earthquake, significant oxygen isotopic anomaly of $+0.24 \%$ in groundwater was observed a few months before the M 6.6 earthquake. On the other hand, there is no anomalous change of the $\delta^{2} \mathrm{H}$ values. At the Kumamoto earthquake, similar $\delta^{\circledR} \mathrm{O}$ anomaly, up to $+0.4 \%$ was observed a few months before the M7.3 earthquake. In addition a large anomaly of $+0.4 \%$ was observed one month after the earthquake. Again there is no anomalous change of the $\delta^{2} \mathrm{H}$ values during the time period. The most probable mechanism to cause $\delta^{\circledR} \mathrm{O}$ enrichment without $\delta^{2} \mathrm{H}$ change is a ${ }^{1} \mathrm{O}$-shift caused by water-rock interaction. In the case of Iceland, both $\delta^{2} \mathrm{H}$ and $\delta^{\circledR} \mathrm{O}$ values of groundwater were changed before and after earthquakes. The difference may be attributable to location specific problem and will be resolved in future research. 